\begin{tabular}{|c|c|c|}
\hline & Int.J.Curr.Microbiol.App.Sci (2021) 10(09): 105-113 & \\
\hline $\begin{array}{l}\text { EXCELLENT } \\
\end{array}$ & $\begin{array}{l}\text { International Journal of Current Microbiology and Applied Sciences } \\
\text { ISSN: 2319-7706 Volume } 10 \text { Number } 09 \text { (2021) } \\
\text { Journal homepage: } \underline{\text { http://www.ijcmas.com }}\end{array}$ & $\$ 9$ \\
\hline $\begin{array}{l}\text { EXCELLENT } \\
\text { PUBLISHERS }\end{array}$ & & www:ijemas.com \\
\hline
\end{tabular}

\title{
Metallo - $\beta$ - Lactamase Producers among Carbapenem Resistant Gram- Negative Isolates from Clinical Samples in a Tertiary Care Hospital
}

\author{
M. Shabnum ${ }^{1} *$ and P. Sreenivasulu $\operatorname{Reddy}^{2}$ \\ ${ }^{1}$ Department of Microbiology, Katuri Medical College, Guntur, \\ Andhra Pradesh - 522019, India \\ ${ }^{2}$ Department of Microbiology, Narayana Medical College, Nellore, \\ Andhra Pradesh - 524003, India \\ *Corresponding author
}

\section{A B S T R A C T}

\section{Keywords}

Gram - Negative

Bacilli,

Carbapenem

Resistance, Metallo

$-\beta$ - lactamase,

Combined Disc

Diffusion Assay,

Imipenem, EDTA

Article Info

Accepted:

10 August 2021

Available Online:

10 September 2021
Gram - Negative Bacilli (GNB) are important cause of UTI, Blood stream infections, hospital acquired pneumonias. With the Carbapenems becoming the drug of choice in treating Multidrug resistant Organisms (MDRO) due to their safety and efficacy, there is rise in Carbapenem Resistant organisms which is becoming a threat to health care setup. Early diagnosis of Metallo - $\beta$ - lactamase (MBL) producers by routine laboratory methods makes it the need of the hour to prevent spread of resistant strains. To detect MBL producers among Carbapenem resistant GNB. GNB were isolated from 2576 various clinical samples received by Department of Microbiology between December 2020 to March 2021. MBL production among Carbapenem resistant GNB was tested by Combined Disc Diffusion Assay using Imipenem disc and Imipenem + EDTA disc. Results: 899 GNB were isolated among 2576 samples with E. coli (35.05\%) followed by Klebsiella species (28.58\%) and Pseudomonas aeruginosa (14.90\%). 180 isolates (20.02\%) were Carbapenem Resistant GNB of which 55 isolates $(30.55 \%)$ were MBL producers with Klebsiella species $(29.01 \%)$ being highest MBL producer followed by Pseudomonas aeruginosa (27.27\%). Rapid dissemination of MBL producers is worrisome making routine detection of MBL strains important. Regular surveillance, strict adherence to infection control measures and implementation of proper antibiotic policy is crucial to minimize the increasing Carbapenem resistance.

\section{Introduction}

Gram-negative bacilli are responsible for causing various infectious diseases such as urinary tract infections (UTIs), pneumonia, septicemia, soft tissue infection, opportunistic infections, and nosocomial infections. ${ }^{[1,2]}$ Carbapenems are broad spectrum antibiotics used for treatment of nosocomial infections caused by gram negative bacilli. Carbapenem 
resistant gram-negative bacilli are an emerging threat to the patient as well as health care system as they are associated with infections leading to high morbidity, mortality prolonging hospital stay and cost with limited options like aminoglycosides, colistin, tigecycline and Fosfomycin. Mainly multidrug resistant gram-negative bacilli comprise of Escherichia coli, Klebsiella pneumoniae, Pseudomonas aeruginosa, Acinetobacter baumannii accounting for $11.5-13.5 \%$ of the Carbapenem resistant infections in the ICU. ${ }^{[3,4,5,6,]}$ Resistance to carbapenems can be due to lack of porin permeability, increase in expression of active efflux pumps, production of metalloenzymes, target site or outer membrane modifications. ${ }^{[7]}$ When such organisms produce Carbapenemases, the outcome for the patient is usually clinical failure, high ICU mortality. ${ }^{[5]}$

$\beta$ - lactams were wonder drugs till $\beta$ lactamases (ESBL and MBL) producing strains started emerging. Metallo - beta lactamases (MBLs) are Carbapenem hydrolyzing enzymes inhibited by metal chelating agents like EDTA ${ }^{[1,6,7]}$ MBL strains are more likely to cause invasive disease and higher hospital case fatality rate compared to other Carbapenem resistant strains. ${ }^{[8,9,10]}$ These organisms carry multidrug resistance genes and only viable treatment options are potentially toxic drugs and are only reserve antibiotics in hospitals. Extensive dissemination of MBL strains among Gram Negative bacilli are responsible for chronicity and relapse of infection leading to high morbidity and mortality, posing a serious health risk to the patients. ${ }^{[1,11,12]}$ Increased mortality rates can be attributed to inadequate empirical therapy and indiscriminate use of antibiotics. $^{[13]}$

Though various recent advances in diagnosis of resistant patterns with molecular detection techniques, conventional methods are economical, reliable for screening and affordable to the patient. ${ }^{[1] .}$ Hence this study was aimed at detecting Carbapenem resistance among gram negative isolates from clinical samples of ICU patients and detect MBL producers among them.

The main objectives of this study includes to isolate gram-negative bacilli from clinical samples. To detect the antibiotic sensitivity pattern of isolated gram-negative bacilli. And also to detect MBL production (Carbapenem Resistance) among the isolated gram-negative bacilli.

\section{Materials and Methods}

\section{Ethical Consideration}

Institutional Ethical Clearance was obtained prior to the study.

\section{Place of study}

Department of Microbiology, Narayana Medical College \& Hospital, Nellore

\section{Source of Clinical Samples}

All Clinical samples received by Microbiology laboratory for culture and sensitivity testing

\section{Study Design}

Prospective study over a period of 4 months (December 2020 - March 2021)

\section{Inclusion Criteria}

Gram negative bacilli isolated from clinical samples.

\section{Exclusion Criteria}

Isolates other than gram negative bacilli 
Isolation of gram-negative bacilli from clinical samples

Various clinical samples received by the Microbiology laboratory for culture and sensitivity testing were inoculated on Nutrient agar, Blood agar and Mac Conkey agar. The culture plates were incubated at $37^{\circ} \mathrm{C}$ for 24 48 hours.

Once the growth was obtained, Gram negative bacilli were isolated based on morphology and gram stain

Detection of the antibiotic sensitivity pattern of gram-negative bacilli

Identification and Antibiotic susceptibility tests were performed by VITEK2 based on Clinical and Laboratory Standards Institute (CLSI) guidelines.

Isolates flagged for Carbapenem resistance were taken for further testing

\section{Detection of MBL production}

The imipenem resistant strains were tested for MBL production by a combined disc diffusion assay using Imipenem disc and Imipenem + EDTA disc

The zone diameter difference of $>5 \mathrm{~mm}$ around the imipenem-EDTA disc in comparison to the zone size of the imipenem disc, were confirmed as MBL producer.

\section{Results and Discussion}

Out of 2576 clinical samples (Urine, Blood, Pus, Swab, Sputum, ET aspirations, ET tube, Tracheal Section, CSF, Body fluids [pleural fluid, pericardial fluid, bile fluid, synovial fluid, drain fluid]) received by the Department of Microbiology, 899 were culture positive for Gram Negative Bacilli.
In the present study out of 2576 clinical samples, 899 isolates were culture positive for Gram negative bacilli with E. coli being the most prevalent pathogen with occurrence of $35.05 \%$ identical to the findings by Dumaru et al., $(38 \%)^{[1]}$ and Fatima et al., (38\%). ${ }^{[14]}$ Higher isolation rates $(54 \%)$ were reported by Maraki et al., ${ }^{[15]}$

Several studies have reported E. coli to be the most commonly isolated Gram-Negative Bacilli causing UTI, Septicemia and other infections. ${ }^{[14,16]}$ Klebsiella (28.58\%) followed by Pseudomonas aeruginosa (14.90\%) were isolated in our study similar to findings reported by Sofia Maraki et al., $(16.5 \%)^{[15]}$ and Yung - Chih Wang et al., $(22.22 \%) \cdot{ }^{[17]} \mathrm{M}$. A. Garbati et al., reported Klebsiella pneumonia to be the most predominant GNB isolated $(52.8 \%)$ followed by E. coli $(22.98 \%)$ and Enterobacter $(20.6 \%) .{ }^{[18]}$

In our study, all the strains were resistant to Ampicillin (100\%) followed by higher resistance to Cefixime (65.6\%), Cephotaxime (65\%) with least resistance being towards Colistin (19.4\%). Similar high resistance to $3^{\text {rd }}$ generation cephalosporins and least resistance to colistin $(0 \%)$ have been reported by Dumaru et al. ${ }^{[1]}$ The reason oh high resistance finding could be widespread use of $3^{\text {rd }}$ generation cephalosporins. These findings are in concordance with findings reported by Kaur et al., (Ceftazidime $-56.67 \%$, Cefoperazone $61.67 \%)^{[19]}$ and Bijayni Behera et al., (Ceftazidime $70 \%)^{[20]} 100 \%$ resistance to cephalosporins was reported by Ding et al., which is alarmingly high. ${ }^{[21]}$ In our study the multidrug resistant strains shared significant sensitivity towards colistin making this as reserve drug for treatment of serious infections. Studies by Bandana Baniya et al., ${ }^{[3]}$ and Koomanachai $\mathrm{P}$ et al., ${ }^{[22]}$ also show promising sensitivity to polymyxin B and colistin. 
In the present study we report 180 isolates among 899 Gram Negative Bacilli (GNB) $(20.02 \%)$ as Carbapenem resistant GNB with highest resistance reported by Citrobacter species $(55.8 \%)$. E. coli, Klebsiella species and Pseudomonas aeruginosa Carbapenem resistance was found as 20\%, $18.2 \%$ and $22.75 \%$ respectively. Similar Carbapenem resistance rates were reported by Maraki et al., $(6.4 \%)^{[15]}$, Mc Conville TH et al., $(11 \%) .^{[23]}$ The majority of carbapenem resistance was reported by Klebsiella pneumoniae (92\%).

M. A. Garbati et al., ${ }^{[18]}$ reported $33.33 \%$ of carbapenem resistant organisms stressing on the emerging carbapenem resistance among the Enterobacteriaceae as a major global public health problem with increase in healthcare casts, treatment failures with added mortality. Highest Carbapenem resistance was reported by $K$. pneumoniae (51.7\%), followed by E. coli $(24.1 \%)$ and Enterobacter (20.7\%).

Carbapenems have remained as last resort antibiotic due to raising ESBL or plasmid mediated AmpC producing organisms.

These pathogens are reported to be resistant to other classes of antibiotics like quinolones and aminoglycosides. With the increasing prevalence of Carbapenem resistance over the past few years, therapeutic options have become limited with colistin and tigecycline to spare as lifesaving antibiotics. ${ }^{[18]}$ Prevalence of Carbapenem resistant Enterobacteriaceae was reported as $24.7 \%, 29.8 \%$ and $37.5 \%$ by $\mathrm{Al}$ - Dhaheri et al., ${ }^{[24]}$, Khorasani et al., ${ }^{[25]}$ and
$\mathrm{Xu}$ et al. ${ }^{[26]}$ respectively. Xu et al. ${ }^{[26]}$ have reported Klebsiella species accounting for maximum CRE (39.3\%) followed by E. coli (22.0\%). In contrast to our study lowest rate of carbapenem resistance was found in Citrobacter (20\%).

Among the mechanisms of Carbapenem resistance in Enterobacteriaceae, the acquisition of specific genes encoding Carbapenemases play a major role which are mostly plasmid mediated and associated with mobile genetic structures like transposons or integrons. This increases the rate of dissemination and spread of Carbapenemases genes. $^{[26,27]}$

Among the 180 carbapenem resistant strains in our study, 55 were MBL producers $(30.55 \%)$. We have reported Klebsiella species to be the highest MBL producers $(29.01 \%)$ followed by Pseudomonas aeruginosa (27.27\%).

Though Dumaru et al., ${ }^{[1]}$ have reported lower MBL producer rates (16.24\%), they have reported Klebsiella species as highest MBL producer $(26.53 \%)$ followed by Pseudomonas $(26.31 \%)$ similar to our findings. Similar rates have been reported by Kaur et al., $(34.8 \%)^{[16]}$, Anuradha et al., $(28.57 \%)^{[28]}$ and Baniya et al., $(22 \%) .{ }^{[3]}$ Similar higher rates of MBL producers were reported by Charan Kaur et al., $(30 \%)^{[19]}$ and Mishra SN et al., $(58.28 \%)^{[13]}$ in concordance with our findings. Lower rates were reported by Yassin NA et al., (12.7\%). ${ }^{[29]}$

Table.1 Gram Negative Isolates among Clinical Samples

\begin{tabular}{|c|c|c|}
\hline & $\mathbf{N}$ & $\mathbf{\%}$ \\
\hline Total samples & 2576 & $100 \%$ \\
\hline GNB & 899 & $34.89 \%$ \\
\hline
\end{tabular}


Table.2 Organism wise Distribution of Gram-Negative Bacilli

\begin{tabular}{|c|c|c|}
\hline GNB & N & \% \\
\hline Escherichia coli & 315 & 35.03 \\
\hline Klebsiella species & 257 & 28.58 \\
\hline Pseudomonas aeruginosa & 134 & 14.90 \\
\hline Acinetobacter baumanii & 48 & 5.33 \\
\hline Citrobacter species & 46 & 5.11 \\
\hline Serratia & 41 & 4.56 \\
\hline Enterobacter & 26 & 2.89 \\
\hline Proteus & 16 & 1.77 \\
\hline Salmonella typhi & 6 & 0.66 \\
\hline Aeromonas species & 6 & 0.66 \\
\hline Providencia species & 1 & 0.11 \\
\hline Chryseobacterium gleum & 1 & 0.11 \\
\hline Total & 899 & 100 \\
\hline
\end{tabular}

Table.3 Resistance Pattern of Gram-Negative Bacilli- Organism wise (\%)

\begin{tabular}{|c|c|c|c|c|c|c|c|c|c|c|}
\hline & $\begin{array}{c}\text { E.coli } \\
(315)\end{array}$ & $\begin{array}{l}\text { Klebsiella } \\
\quad(257)\end{array}$ & $\begin{array}{c}\text { Pseudomonas } \\
\text { (134) }\end{array}$ & $\begin{array}{c}\text { Acineto- } \\
\text { bacter }(48)\end{array}$ & $\begin{array}{c}\text { Citro- } \\
\text { bacter } \\
(46)\end{array}$ & $\begin{array}{l}\text { Serratia } \\
\quad(41)\end{array}$ & $\begin{array}{c}\text { Entero- } \\
\text { bacter } \\
(26)\end{array}$ & $\begin{array}{l}\text { Proteus } \\
\text { (16) }\end{array}$ & $\begin{array}{l}\text { S. typhi } \\
\text { (6) }\end{array}$ & $\begin{array}{c}\text { Aeromonas } \\
\text { (6) }\end{array}$ \\
\hline Amp & 100 & 100 & NA & 100 & 100 & 100 & 100 & 100 & 100 & NA \\
\hline CFX & 49.2 & 66.9 & NA & 83.8 & 100 & 36.6 & 54.5 & 75 & 50 & NA \\
\hline CTX & 68.3 & 84.2 & NA & 72.7 & 68.4 & 31.7 & 63.6 & 66.7 & 50 & NA \\
\hline CFX & 67.9 & 94.7 & NA & 73 & 94.7 & 34.1 & 63.6 & 66.7 & 33.33 & NA \\
\hline CPM & 62.5 & 66.7 & 41.4 & 70.3 & 63.2 & 34.1 & 63.6 & 66.7 & 66.66 & 33.3 \\
\hline COT & 65.1 & 56.1 & 61.6 & 72.7 & 47.4 & 14.6 & 72.7 & 75 & 0 & 33.3 \\
\hline CIP & 73.3 & 45.6 & 40.4 & 62.2 & 47.4 & 17.1 & 68.2 & 100 & 16.66 & 66.7 \\
\hline GEN & 32.4 & 31.07 & 30.3 & 62.2 & 68.4 & 26.8 & 40.9 & 50 & 100 & 16.7 \\
\hline AMK & 23.2 & 35 & 27.3 & 59.5 & 52.6 & 24.4 & 45.5 & 33.33 & 100 & 16.7 \\
\hline TIGE & 13.3 & 47.6 & NA & 37.8 & 52.6 & 22.9 & 45.5 & 75 & 0 & NA \\
\hline $\begin{array}{c}\text { Amox/ } \\
\text { Clav }\end{array}$ & 57.8 & 60.7 & NA & 75.7 & 94.7 & 63.4 & 72.7 & 66.7 & 0 & NA \\
\hline Cef/Sulb & 52.7 & 45.2 & 50.5 & 70.3 & 89.7 & 19.5 & 77.3 & 66.7 & 0 & 33.3 \\
\hline PIT & 38.4 & 57.1 & 23.2 & 67.6 & 63.2 & 26.8 & 50 & 33.33 & 0 & 16.7 \\
\hline IMP & 39 & 33.9 & 27.3 & 56.8 & 57.9 & 24.4 & 31.8 & 33.33 & 0 & 16.7 \\
\hline MRPM & 23.2 & 42.9 & 18.2 & 65.2 & 64.47 & 29.3 & 31.8 & 66.7 & 0 & 16.7 \\
\hline Colistin & 9.8 & 10.5 & 25.3 & 29.7 & 31.6 & 100 & 31.8 & 50 & 0 & 50 \\
\hline CZM & NA & NA & 69.7 & NA & NA & NA & NA & NA & NA & 66.7 \\
\hline LEVO & NA & NA & 99 & NA & NA & NA & NA & NA & NA & 0 \\
\hline
\end{tabular}

Amp: Ampicillin; CFX: Cefoxitin, CTX: Cephotaxime; CFX: Cefixime; CPM: Cefepime; COT: Cotrimoxazole; CIP: Ciprofloxacin; GEN: Gentamicin; AMK: Amikacin; TIGE: Tigecycline; Amox/ Clav: Amoxicillin Clavulanic acid; Cef/ Sulb: Cefaperazone / Sulbactam; PIT: Piepracillin/ Tazobactam; IMP: Imipenem; MRPM: Meropenem; CZM: Ceftazidime; LEVO: Levofloxacin 
Table.4 Resistance of Gram - Negative Bacilli

\begin{tabular}{|c|c|}
\hline Antibiotic & \% \\
\hline Ampicillin & 100 \\
\hline Cefoxitin & 58.3 \\
\hline Cephotaxime & 65 \\
\hline Cefixime & 65.6 \\
\hline Cefepime & 57.4 \\
\hline Cotrimixazole & 58.1 \\
\hline Ciprofloxacin & 58 \\
\hline Gentamicin & 36.7 \\
\hline Amikacin & 31.7 \\
\hline Tigecycline & 28.6 \\
\hline Amox/Clav & 61.9 \\
\hline Cef / Sulb & 54.9 \\
\hline PIT & 40.7 \\
\hline Imipenem & 38.1 \\
\hline Meropenem & 28.5 \\
\hline Colisitin & 19.4 \\
\hline
\end{tabular}

Table.5 Carbapenem Resistant Strains Distribution - Organism Wise

\begin{tabular}{|c|c|}
\hline Organism & $\%$ \\
\hline Citrobacter species & 55.8 \\
\hline Proteus species & 50 \\
\hline Pseudomonas aeruginosa & 22.75 \\
\hline Klebsiella species & 20.8 \\
\hline E. coli & 20 \\
\hline Enterobacter species & 18.2 \\
\hline Aeromonas species & 16.7 \\
\hline Serratia & 2.4 \\
\hline
\end{tabular}

Table.6 Carbapenem Resistance Distribution: Sample wise

\begin{tabular}{|c|c|}
\hline Clinical Sample & $\%$ \\
\hline Urine & 16.4 \\
\hline Tracheal Section & 40 \\
\hline Swab & 14.7 \\
\hline Pus & 25.5 \\
\hline Blood & 9.1 \\
\hline Sputum & 14.7 \\
\hline ET tube / Aspiration & 45 \\
\hline CSF & 50 \\
\hline Body fluids & 50.77 \\
\hline
\end{tabular}


Table.7 MBL producers

\begin{tabular}{|c|c|c|}
\hline Organism & N & \% \\
\hline Klebsiella species & 16 & 29.01 \\
\hline Pseudomonas aeruginosa & 15 & 27.27 \\
\hline Acinetobacter species & 14 & 25.45 \\
\hline E. coli & 9 & 16.36 \\
\hline Enterobacter & 1 & 1.81 \\
\hline Total & 55 & 100 \\
\hline
\end{tabular}

Such variations in the detection rates of MBLs can be attributed to various factors like geographical distribution, infection control practices, methods used to detect MBLs. ${ }^{[8]}$ Pseudomonas has been reported High MBL producer by Yassin et al., $(12.7 \%)^{[29]}$, Mishra SN et al., $(58.28 \%)^{[13]}$, Vinita Choudhary et al., $(20 \%)^{[8]}$ and Dardi Charan Kaur et al., $(30 \%)^{[19]}$. Baniya et al., ${ }^{[3]}$ reported highest MBL production among Acinetobacter species $(22 \%)$.

Such disparity in the detection rates and the predominant MBL producing organism brings out the importance and the need for epidemiological surveillance and early diagnostic method for rapid identification of MBL producers, since dissemination of MBL producers poses a therapeutic challenge to treating clinicians as it can hydrolyze carbapenems which are being given as best therapy for invasive diseases, critically ill patients, nosocomial infections. ${ }^{[29]}$

Hence such early rapid detection of MBL producers in a routine laboratory can ensure optimal patient care and also timely introduction of appropriate infection control measures to curtail the dissemination of MBL producers further ultimately improving the quality of patient care in health care setup.

Carbapenem Resistance has enormous therapeutic implications as well as important in regards to infection control as such strains are responsible for rapid intra institutional spread. MBL strains develop mutations and participate in horizontal MBL gene transfer with other pathogens making early detection and timely implementation of strict infection control practices. Though PCR is highly accurate and reliable method for MBL detection, it is limited to reference laboratories. Early detection in routine laboratory using conventional methods could help avoid treatment failure.

Monitoring of resistant patterns, drafting antibiotic policy and guidelines should be implemented for ultimate better patient management. Regular antimicrobial susceptibility surveillance is essential. Continuous effort to contain drug resistance and MDR organisms is highly required to preserve the effectiveness of antibiotics and prevent medical progress to go back to preantibiotic era. ${ }^{[30]}$

\section{References}

1. Rabina Dumaru, Ratna Baral and Lok Bahadur Shrestha. Study of Biofilm formation and Antibiotic resistance pattern of Gram-Negative bacilli among the clinical isolates at BPKIHS, Dharan. BMC Res Notes (2019) 12:38: $1-6$

2. Andhale, J. D. et al., Incidence of Pseudomonas aeruginosa with special reference to drug resistance and biofilm formation form clinical samples in tertiary care hospital. J Pharm Biomed Sci; Vol 06 (6): 387 $-391$

3. Bandana Baniya et al., Biofilm and Metallo beta - lactamase production among the strains 
of Pseudomonas aeruginosa and Acinetobacter spp at a tertiary care hospital in Kathmandu, Nepal. Ann ClinMicrobiolAntimicrob (2017) 16:70:1-4

A. Xu, B. Zheng, Y. C. Xu, Z. G. Huang, N. S. Zhong, C. Zhuo. National Epidemiology of Carbapenem - resistant and extensively drug resistant Gram - Negative bacteria isolated from blood samples in China in 2013. Clin Microbiol Infect 2016; 22: S1 - S8

4. Kontopidou F et al., infections caused by Carbapenem - resistant Klebsiella pneumoniae among patients in Intensive Care Units in Greece: a Multi - center study on clinical outcome and therapeutic option. Clin Microbiol Infect 2016; 20: O117 - O123

5. Nepal K, Pant N D, Neupae B, Belbase A, Baidhya R, Shreshta R K et al., Extended Spectrum beta - lactamase and Metallo beta lactamase production among Escherichia coli and Klebsiella pneumoniae isolated from different clinical samples in a tertiary care hospital in Kathmandu, Nepal. Ann Clin Microbiol Antimcrob. 2017; 16:62

6. Saffari M, Firoozeh F, Pourbabaee M, Zibaei M. Evaluation of Metallo - beta - lactamase production and carriage of bla-VIM Ges in Pseudomonas aeruginosa isolated from burn wound infections in Isfahan. Arch Trauma Res. 2016; 5:4

7. Choudhary V, Pal N, Hooja S. Prevalance and antibiotic resistance pattern of Metallo - beta lactamase producing Pseudomonas aeruginosa Isolates from Clinical specimens in a Tertiary Care Hospital. J Mahatma Gandhi Inst Med Sci 2019; 24:19-22

8. Dahiya S, Singla P, Chaudhary U, Singh B. Carbapenemases: A review. Int J Adv Health Sci $2015 ; 2: 11-7$

9. Ranjan S, Banashankari G, Babu P S. Comparison of epidemiological and antibiotic susceptibility pattern of Metallo - beta lactamase positive and Metallo - beta lactamase negative strains of Pseudomonas aeruginosa. J Lab Physicians 2014; 6: 109 - 13

10. Costerton J W, Stewart P S, Greenberg E P. Bacterial biofilms: a common cause of persistent infections. Science. 1992;284 (5418): $1318-22$

11. Jr C J S, Mende K, Beckius M L, Akers K S, Romano D R, Wenke J C, et al., Biofilm formation by clinical isolates and the implications in chronic infections. BMC Infect Dis. 2013; 13-47

12. Mishra S N, Biswal S R, B Behra B K, Pattnaik D. Detection of prevalence of Metallo - beta lactamases in clinical isolates of imipenem resistant Pseudomonas aeruginosa from neonatal septicemia cases in a tertiary hospital in Odisha, India. Int J ContempPediatr 2018; 5: $61-6$

13. Fatima S, Prashanthi K, Nagamani K. Comparitive evaluation of biofilm production in Multidrug resistant and sensitive Gram Negative clinical isolates. Int J Curr Microbiol Appl Sci. 2015; 4(6): 918 - 26

14. Sofia Maraki, George Samonis, Drosos E Karageorgopoulos, Micheal N. Mavros, Diamantis Kofferidis, Mathew E. Falagas. Invitro Antimicrobial Susceptibility to Isepamicin of 6296 Enterobacteriaceae Clinical Isolates collected at a Tertiary Care University Hospital in Greece. Antimicrobial Agents and Chemotherapy. 2021; 56(6): 3067 - 3073

15. Kaur N, Kaur A, Singh S, Prevalence of ESBL and MBL producing Gram Negative isolates from various clinical samples in a tertiary care hospital. Int J Curr Microbiol Appl Sci 2017; 6(4): $1423-30$

16. Yung - Chih Wang, Ya - Sung Yang, Ti - Yin, Shu Chen Kuo, Jung - Chung Lin, Feng - Yee Chang. Comparison of Susceptibility of Enterobacteriaceae causing Community Onset Urinary Tract Infection to Isepamicin and Amikacin by the disc Diffusion Method. J Med Sci J Med Sci 2014; 34 (6): 241 - 246

17. M. A. Garbati, H. Sakkijha, A Abushaheen. Infections due to Carbapenem Resistant Enterobacteriaceae among Saudi Arabian Hospitalized Patients: A Matched Case Control Study. BioMed Research International. 2016; ID. $\quad 3961684: \quad 1 \quad-\quad 9$. https://dx.doi.org/10.1155//2016/3961684

18. Dardi Charan Kaur, Dr. Wankhede S. V. A study of biofilm formation \& Metallo $-\beta-$ Lactamases in Pseudomonas aeruginosa in a tertiary care rural hospital. International Journal of Scientific and Research Publications. Oct 2013; 3(10): 1- 7

19. Bijayini Behera, Anupam Das, Purva Mathur, Arti Kapil. High Prevalence of Carbapenem Resistant Pseudomonas aeruginosa at a tertiary 
care centre of North India. Are we under reporting? Indian J Med Res 128, September 2008. $324-325$

20. Yijun Ding, Yajuan Wang, Yingfen Hsia, Mike Sharland, Paul T. Heath. Systematic review of Carbapenem - resistant Enterobacteriaceae causing neonatal sepsis in China. Ann Clin Microbiol Antimicrob (2019); 18: 36: 1 - 8

21. Koomanachai $\mathrm{P}$, Tiengrim $\mathrm{S}$, Kiratisin $\mathrm{P}$, Thamlikitkul V. Efficacy and safety of Colistin (Colistimethate sodium) for therapy of infections caused by multidrug resistant Pseudomonas aeruginosa and Acinetobacter baumanii in Siriraj Hospital, Bangkok, Thailand. Int J Infect Dis. 2007; 11(5): $402-6$

22. Mc Conville T H, Sullivan S B, Gomez Simmonds A, Whittier S, Uhlemann A - C (2017). Carbapenem - resistant Enterobacteriaceae colonization (CRE) and subsequent risk of infection and 90-day mortality in critically ill patients; an observational study. PLoS ONE 12 (10): e0186195.

https://doi.org/10.1371/journal.pone.0186195

23. Al - Dhaheri A S, Al - Niyadi M S, Al Dhaheri A D et al., Resistance patterns of bacterial isolates to antimicrobials from 3 hospitals in the United Arab Emirates. Saudi Med J 2009; 30: 618 - 23

24. Khorasani G, Salehifar E, Eslani G. Profile of microorganisms and antimicrobial resistance at a tertiary care referral burn centre in Iran: emergence of Citrobacter freundii as a common microorganism. Burns 2008; 34: 947 52

25. Yanding $\mathrm{Xu}$, Bing $\mathrm{Gu}$, Mao Huang, Haiyan Liu, Ting $\mathrm{Xu}$, Wenying Xia, Tong Wang. Epidemiology of Carbapenem resistant Enterobacteriaceae (CRE) during 2000 - 2012 in Asia. J Thorac Dis 2015; 7(3): 376 - 385.

26. Cuzon G, Naas T, Nordmann P. Functional characterization of Tn 4401, a Tn 3 - based transposon involved in blaKPC gene mobilization. Antimicrob Agents Chemother 2011; 55: $5370-73$

27. De Anuradha $S$, Kumar S, Baveja S M. Prevalence of metallo-beta-lactamase producing Pseudomonas aeruginosa and Acinetobacter species in intensive care unit in a tertiary care hospital. Indian J Crit Care Med. 2010; $\quad$ 14(4): $217 \quad-\quad 9$. https://doi.org/10.4103/0972 - 5229. 76089

28. Yassin N A, Khali H M, Hassan A O. Prevalence of Metallo - $\beta-$ lactamase producing Pseudomonas aeruginosa in wound infections in Duhok city, Iraq. Int J Res Med Sci 2014; 2:1576 - 9

29. Shabnum M, P. Sreenivasulu Reddy and Vasundhara, P. 2018. Common Isolates among Suspected Cases of Septicemia with a special Emphasis on Multidrug Resistant Strains. Int J. Curr. Microbiol. App. Sci. 7(03): 711 - 721. doi:

https://doi.org/10.20546/ijcmas.2018.703.083

\section{How to cite this article:}

Shabnum, M. and Sreenivasulu Reddy, P. 2021. Metallo- $\beta$ - Lactamase Producers among Carbapenem Resistant Gram-Negative Isolates from Clinical Samples in A Tertiary Care Hospital. Int.J.Curr.Microbiol.App.Sci. 10(09): 105-113. doi: https://doi.org/10.20546/ijcmas.2021.1009.012 